\title{
Microrheometry of Sub-Nanoliter Biopolymer Samples: Non-Newtonian Flow Phenomena of Carnivorous Plant Mucilage
}

\author{
Philipp Erni $^{a}$, Matthieu Varagnat ${ }^{a}$, Christian Clasen $^{b}$, Jérôme Crest ${ }^{a}$, Gareth H. McKinley ${ }^{a *}$ \\ ${ }^{a}$ Hatsopoulos Microfluids Laboratory, Massachusetts Institute of Technology, \\ 77 Massachusetts Ave., Cambridge MA 02139 and \\ ${ }^{b}$ Department of Chemical Engineering, Katholieke Universiteit Leuven, 3001 Heverlee, Belgium
}

\begin{abstract}
Sundew plants (Drosera) capture insects using tiny drops of a viscoelastic fluid. These mucilage droplets are typically tens of micrometers in diameter, corresponding to fluid volumes in, or below, the nanoliter range. In contrast to other carnivorous plants, the physical principles and the role of rheology in the capturing mechanism are not yet fully understood. The rather simple chemical composition reported for the capturing fluid (a high molecular weight acidic polysaccharide composed of a D-glucurono-D-mannan backbone with alternating monosaccharide sidegroups) is in stark contrast to the compositional and structural complexity of other biological materials with strong extensional responses, including fibers, silks, or mucins. Here we show that the strategy used by the sundew plant combines the effects of high fluid viscosity, extensional viscoelasticity, capillary thinning and a liquid-to-solid transition driven by solvent mass transfer and aided by extensional strain-stiffening. More generally, we demonstrate how the rheological material functions of microscopic biopolymer samples in the sub-nanoliter range can be measured using an interferometry-based microrheometer for shear deformations and a capillary thinning microrheometer for extensional deformations.
\end{abstract}

$\overline{* e-m a i l:}$ gareth@mit.edu 


\section{INTRODUCTION}

Drosera ('sundew plants') are carnivorous plants; they capture insects using tiny drops of a viscoelastic fluid secreted by stalked glands on their leaf laminae (Fig. 1) [1,2]. These mucilage droplets are typically tens of micrometers in diameter, corresponding to fluid volumes in or below the nanoliter range. Insects get trapped by the sticky liquid, causing inflection of the tentacles [2], and a metabolic cascade is then initiated on the leaf surface, gradually digesting the prey, as already described by Darwin in 1875 who noted "The fact that a plant should secrete, when properly excited, a fluid containing an acid and ferment, closely analogous to the digestive fluid of an animal, was certainly a remarkable discovery" [1]. A second type of sessile gland on the leaves secretes digestive enzymes, transforming the nutrients into a solution which is then taken up by the plant. The response of the mucilaginous glands appears to depend on the chemical nature of the stimulus surface $[1,2]$ : it is absent for water droplets or glass, whereas the glands respond strongly in the presence of nitrogen, for example with protein-containing stimuli (such as insects).

Drosera mucilage contains an acidic polysaccharide as the only high molecular weight compound [2]. Its backbone is composed of alternating D-glucuronic acid and D-mannose units, both present in their pyranose forms, plus monosaccharide side groups drawn from the group of L-arabinose, D-xylose and D-galactose, with total chain molecular weights in the range $M_{r} \approx 2 \cdot 10^{6}-5 \cdot 10^{6} \mathrm{~g} \cdot \mathrm{mol}^{-1}$; the known repeat units are given in Fig. 1e. The secreted fluid is reported to be an approximately 4 wt.-\% aqueous solution of this polysaccharide, with only minute quantities of free monosaccharides present. In the native solution at $\mathrm{pH} 5$, most of the acidic residues are neutralized with bivalent counterions (mostly $\mathrm{Ca}^{2+}$ and $\mathrm{Mg}^{2+}$ ); the isoelectric point of the polysaccharide is at $\mathrm{pH} \approx 3$. Polysaccharides from different Drosera species have been reported to be similar in structure and composition [2].

Trapping strategies have recently been investigated for various carnivorous plants. For example, a mechanical snap-through instability coupled with biochemical signaling is used by the Venus fly trap (Dionaea 
muscipula) [3, 4]). The pitcher plant (Nepenthes [5]) uses a 'toilet bowl' concept marrying the effects of fluid viscoelasticity with surface lubricity. In the latter example, insects are captured with a smooth, slippery surface that lets the prey slide into a pool of capturing fluid that has a large extensional viscosity.

Unlike the relatively large fluid volumes ( $m l$ volumes) produced by other carnivorous plants [5], the volumes of the mucilage droplets secreted by Drosera are only of the order of nanoliters (see Fig. 1 for an example). The exceedingly small drop sizes, along with the high extensional viscosities, make bulk rheological characterization using traditional instruments impossible. Although recent microrheometrical methods based on particle tracking $[6,7]$ can be very powerful for measuring and spatially resolving the local viscoelasticity of biofluids, the required sample volumes are still orders of magnitude larger than the mucilage droplets, and the validity of the generalized Stokes-Einstein equation on which the analysis is based is limited to small strain linear viscoelasticity.

Adding to the challenge, microrheometric techniques typically involve actively-actuated microprobes $[8,9]$ or passive measurements on microparticles [6], intended to reveal rheological information on small length scales $[7,10,11]$. Unfortunately, immersing even a small number of tracer micro- or nanoparticles into a nanoliter droplet of a viscoelastic polymer solution is experimentally challenging. Additionally, the enormous viscosity levels found in the mucilage studied here are problematic for current particle tracking methods, due to limitations in the applicable force scales and the spatial resolution of the detection optics. On the other hand, several micromechanical tensile tests have been developed recently and applied, for example, to biological attachment systems such as the Gecko foot adhesive mechanism [12] and for quantifying the adhesive properties of spider silk [13]. In another approach, termed 'capillary micromechanics', the compressional and shear moduli of soft particles were recently quantified using flows through tapered glass capillaries $[14,15]$. Until now those methods have mostly been used for tests on predominantly solid materials, but not for measurements of the extensional viscoelasticity of complex fluids. 
Here, we demonstrate how the extensional rheological material functions of very small volumes of biopolymer fluids can be measured using a simple capillary thinning microrheometer; this custom-built instrument is based on microscope video imaging of the surface-tension driven drainage from a slender liquid filament [16]. We use these experiments to study the rheology and non-Newtonian flow behavior of mucilage drops formed by Drosera plants by performing extensional microrheometry in situ with drops retained on the original plant leaves. In addition, the evolution of elastocapillary instabilities observed on mucilage filaments are investigated, and we discuss the morphological characteristics of the residual biopolymer fibers. These experiments provide insight into the biophysical principles behind the capture mechanisms in Drosera plants; moreover, the results also suggest that the fluid studied here is of interest not only for the study of biological fibrils, but also as a model material for the synthesis and processing of biodegradable fibers, for example those produced by electrospinning.

\section{EXPERIMENTAL SECTION}

\subsection{Materials}

Sundew plants (Drosera spathulata) were purchased from Hirt's Gardens (Medina, Ohio). The plants are grown in acrylic glass containers kept at a relative humidity $\varphi=0.3 \pm 0.05$ and fed with insects sporadically. Trimethylsiloxy-terminated polydimethylsiloxane (DMS-T56, Gelest Inc., Tullytown PA) is used as a reference fluid for calibration of the microrheometers used in this study. Relative humidities are measured with a miniaturized hygrometer (Speedtech SM-28 Skymaster). 


\subsection{Capillary Thinning Microrheometry}

Capillary breakup extensional rheometry (or the 'caber' technique) [17-20] is an important technique for measurements of the apparent transient extensional viscosity of polymer solutions, paints, inks, and other complex fluids [19]. A standard experiment involves the formation of a liquid bridge of the test fluid between two cylindrical endplates. Applying a step strain and separating the endplates in the axial direction results in the formation of an elongated liquid filament that is beyond its static Rayleigh-Plateau stability limit. The shape of the free surface subsequently evolves under the action of the capillary pressure, which acts as a driving force for the thinning of the filament [21]. This surface tension-driven flow is resisted by a combination of viscous and elastic stresses in the fluid [17-19, 22]. For a material to be amenable to microfilament rheometry, the viscocapillary time scale $t_{v c}=\eta_{0} R_{0} / \sigma$ (the ratio of the zero shear viscosity $\eta_{0}$ to the capillary pressure $\sigma / R_{0}$, where $\sigma$ is the surface tension and $R_{0}$ the radius of the filament) must be sufficiently long that a well-defined capillary-driven flow is established: capillary thinning must occur at time scales compatible with the temporal resolution of a laser micrometer or a video camera, while fluid inertia should be negligible $[19,23,24]$. High viscosity levels are therefore beneficial for establishing a smooth capillary thinning flow that is slow enough to enable extensional rheometry. Fluid inertia is important for low viscosity fluids, such as dilute polymer solutions, but can be neglected for high values of the Ohnesorge number $O h=t_{v c} / t_{r}=\eta_{0} / \sqrt{\rho R_{0} \sigma}>1$, a dimensionless group comparing the viscocapillary and the Rayleigh time scale $t_{r}=\left(R_{0}^{3} \rho / \sigma\right)^{1 / 2}$ [25]. Small sample diameters and high fluid viscosities both help to minimize inertia effects the importance of inertia in microfilament extensional rheometry [26, 27].

In commercially available instruments traditional caber experiments, the time-dependent midfilament radius $R_{\text {mid }}(t)$ of the liquid bridge is measured using a laser micrometer and the global evolution in the filament profile $R(z, t)$ is recorded using macrophotography or high speed digital video imaging. An experimental data set consists of a time series of $R_{\text {mid }}(t)$ which is then $R_{\text {mid }}(t)$ 
is then analyzed further based on appropriate fluid models. For the experiments described here, we have instead built a microscope-based capillary thinning extensional rheometer (Fig. 2) In f m based on direct analysis of video microscopy images in conjunction with image analysis and an edge detection procedure. The bottom plate containing a droplet of the sample fluid is mounted on an $x y z$ stage (TXS, Melles Griot) and is brought into contact with the other plate. Liquid bridges are then formed by stretching with the linear translation stage. In the present work, We use precision-cut copper wires and polymer fibers are used as end plates, covering a wide range of possible diameters.

For the in-situ experiments on the mucilage drops, a single leaf is mounted onto the $x y z$ stage with double-sided adhesive tape. A CCD-based digital video camera (mvBlueFOX, Matrix Vision GmbH, Germany) is used to capture movies with a frame rate of $60 \mathrm{~Hz}$. We use the Matlab Image Processing Toolbox (The Mathworks; http://www.mathworks.com) to extract the full surface profile from the movies (Fig. 2b). Briefly, the processing steps involve (i) Sobel filtering and edge detection on each frame, resulting in a black and white image; (ii) boundary tracing on both sides of the image; and (iii) determination of the minimum diameter or, alternatively, the diameter in a selected cross-sectional plane (either the midfilament plane, or in the plane corresponding to the smallest diameter after the fiber has dried out completely). For the in-situ experiments, we choose the $z$ position at which the radius is evaluated such that it is located at the thinnest point at the end of the experiment (i.e. by minimizing $R(z, t)$ for $t \rightarrow \infty$ ). The radius $R_{m}(t)$ is then measured at this $z$ position for all frames (see Figs. 4a and 5a for examples, where the measuring position is indicated by the dashed line). In the case of a symmetrical liquid bridge (Fig. 4a), the minimum radius $R_{m}$ is located in the midplane and is identical to $R_{\text {mid }}$ as measured in traditional caber experiments. Notice that a solid thread formed from a drying polymer solution by extensional deformation is not to be confused with a statically stable liquid bridge [28]. For eapillary thinning experiments performed at elevated humidity, 
the entire fixture is kept under a steady stream of conditioned air. The main stream is separated from the sample with an additional microscope slide to minimize convection in the region of the filament thread and to avoid liquid condensation on the camera lens; the miniature humidity probe is placed $5 \mathrm{~mm}$ away from the sample.

The error due to the image analysis method used is small compared to variations inherent to native biomaterials (including variations between individual and between species as well as environmental variations during growth and mucilage formation). For individual images, the maximum absolute error $\Delta R$ expected for the radius measurement is typically in the range of $\Delta R \approx \pm 0.5-1.2 \mu \mathrm{m}$. Since large numbers of images are acquired and analyzed for the measurements, the mean absolute variation remains relatively small. For example, a mean absolute variation of $0.35 \mu \mathrm{m}$ amounts to about $3 \%$ of the largest radius to less than $10 \%$ of the smallest radius measured in Fig. 5.

\subsection{Shear Microrheometry}

To measure the steady shear viscosity, we use a miniaturized, strain-controlled sliding plate rheometer, the Flexure-based Microgap Rheometer (FMR) [29]. It is based on a precision-machined compound flexure design, consisting of pairs of double leaf springs arranged within a shared translating frame. This ensures purely translational motion up to large deformations while maintaining precision parallel alignment of the shear cell. A plane Couette shear flow is generated between two optical interferometry flats (Melles Griot) which are used as the shearing surfaces and are polished to within $30 \mathrm{~nm}$ over a $25 \mathrm{~mm}^{2}$ surface, with a semireflective $\mathrm{TiO}_{2}$ coating of $100 \mathrm{~nm}$ thickness. The bottom part of the frame is used to translate the actuating plate, and is driven by an inchworm-style nanomotor with a maximum displacement of $1.6 \mathrm{~mm}$, a step resolution of $0.5 \mathrm{~nm}$ and a maximum speed of $2 \mathrm{~mm} \cdot \mathrm{s}^{-1}$. The top leaf springs are used to detect the shear stress with a noninvasive inductive proximity sensor. Shear stresses are calculated after calibrating the 
detection springs according to the beam relationship, $K=E \cdot\left(w b^{3} L^{-3}\right)$, where $K$ is the spring constant of the flexure, $L$ is its length, $w$ is its depth, $b$ the thickness and $E$ the Young's modulus of the metal (aluminium). The available measuring range and its dependence on the geometry of the compound flexure and the surface area of the shear cell have been discussed previously by Clasen et al. [29].

Each glass plate is mounted on a three-point nanopositioning stage (New Focus, San Jose, CA). For measurements with the plant mucilage, a measuring gap of $12 \mu \mathrm{m}$ and a shearing surface of $25 \mathrm{~mm}^{2}$ is used. Because the required volume $V \approx 0.3 \mathrm{nl}$ is still above the available volume of a single mucilage drop, fluid is introduced onto the bottom interferometry plate by spreading multiple drops directly from the plant leaves. To provide a controlled atmosphere and suppress sample drying during the loading process and during the experiment, the rheometer fixture is kept under a curtain of conditioned air (temperature of $23{ }^{\circ} \mathrm{C}$ and a relative humidity of $\varphi=0.8$ ) continuously flowing from a tube mounted above the shear cell.

\section{RESULTS}

\subsection{Shear and Extensional Microrheometry of Drosera Mucilage}

We first demonstrate how the rheological material functions of nanoliter volume, high viscosity, samples of complex fluids can be measured by shear microrheometry in plane Couette flow and by capillary thinning microrheometry in extensional deformations. The steady shear viscosity $\eta(\dot{\gamma})$ of the mucilage as a function of the shear rate is shown in Fig. 3. The values are of the order $10^{2} \mathrm{~Pa} \cdot s$, with shear-thinning behavior setting in around a eritical shear rate $\dot{\gamma} \approx 0.2 \mathrm{~s}^{-1}$. We use the Carreau-Yasuda constitutive equation [30], $\eta=\eta_{0}\left[1+\left(\dot{\gamma} / \dot{\gamma}^{*}\right)^{a}\right]^{(n-1) / a}$, which is one of the simplest phenomenological models to capture the important features of this rheological response. From a nonlinear least square fit, we obtain a zero shear viscosity $\eta_{0}=122 \mathrm{~Pa} \cdot \mathrm{s}$ and characteristic time scale for shear flow 
$\lambda_{c}=1 / \dot{\gamma}^{*} \approx 0.4 \mathrm{~s}$ (indicating the rate of transition between the plateau viscosity and the shear thinning regime) along with a power-law exponent $n \approx 0.56$ and Carreau parameter $a \approx 0.96$. For reference, the steady shear viscosity of a standard polydimethylsiloxane (PDMS) silicone fluid is included in Fig. 3; the lower Newtonian plateau with $\eta_{0}=605 P a \cdot s$ extends up to a shear rate of $\dot{\gamma} \approx 2 s^{-1}$.

In Fig. 4 a reference capillary thinning experiment performed with the polydimethylsiloxane oil is shown. Due to the high viscosity level, the critical time scale for thinning and breakup is in the range of a few seconds, $t_{c}=14.1 \eta_{0} R_{0} / \sigma \approx 6.9 \mathrm{~s}$, where the numerical front factor is obtained from Papageorgiou's similarity solution for Newtonian fluids undergoing capillary thinning [19, 31]:

$$
\frac{R}{R_{0}}=0.0709 \frac{\sigma}{\eta_{0}}\left(t_{c}-t\right)
$$

The slender filament of PDMS thins and eventually breaks up following a typical Newtonian fluid behavior, with the radius decreasing linearly with time, as predicted by the theory [31] (solid line in Fig. 4).

In Fig. 5(a) a capillary thinning experiment is shown with a single mucilage drop retained on an intact secretory gland. We form a liquid bridge with a single drop still located on the leaf, wetting the side face of a microscope slide. After the drop has wetted the glass surface, the tentacle is retracted to form a liquid bridge with an initial extensional pre-strain of $\ln \left(L_{f} / L_{0}\right) \approx 1.1$ and the subsequent evolution of the filament radius is tracked over time. Here, $L_{0}$ is the length of the initial configuration and $L_{f}$ is the length of the sample after it has been stretched to a predefined strain beyond the Plateau stability limit of the liquid bridge [19]. Following an initial decrease characteristic of viscous liquids, the rate of thinning $d R_{m} / d t$ gradually decreases and eventually the radius converges to a stationary value. This behavior is characteristic for fluids with simultaneous strain-stiffening and evaporative mass transfer of solvent out of the filament into the surroundings [32, 33], and has been observed in other fiber-forming biopolymer solutions, most notably for spider silk [16]. 
Instead of assuming a fluid model a priori for the mucilage, we can calculate directly an apparent extensional viscosity

$$
\eta_{E}(t)=\frac{\Delta \tau}{\dot{\epsilon}}=\frac{\sigma}{-2 \frac{d R_{m}(t)}{d t}}
$$

based on the ratio of the surface tension and the apparent instantaneous strain rate. Here, the tensile stress difference $\Delta \tau$ is obtained from a force balance involving the capillary pressure, and the extensional strain rate $\dot{\epsilon}=-\frac{2}{R} \frac{d R(t)}{d t}=-2 \frac{d \ln \left[R(t) / R_{0}\right]}{d t}$ is derived from the kinematics of the thinning slender filament $[16,18$, 32]

The quantitative description of the transient fluid dynamics associated with the necking process for a complex fluid remains a challenge for capillary thinning rheometry, even in the absence of mass transfer [19]. The time-evolution of the material functions and of the kinematics, in particular the extension rate, has been discussed elsewhere $[17,19,23,34]$. Based on these previous investigations, we use the apparent Hencky strain $\epsilon=\int_{0}^{t} \dot{\epsilon}\left(t^{\prime}\right) d t^{\prime}$ accumulated by a material element in the neck to characterize the fluid deformation. In Fig. 5c the apparent extensional viscosity $\eta_{E}$ evaluated using eq. 2 is plotted as a function of the corresponding Hencky strain. The extensional viscosity $\eta_{E}$ diverges towards the end of the experiment, indicating effective solidification of the fiber; pulling the fiber to higher strains causes brittle failure rather than further extensional deformation. The extensional viscosity should be three times the zero shear viscosity in the low strain limit [35] and we find good agreement with steady shear experiments, where weakly shear thinning behavior with a zero shear viscosity $\eta_{0} \approx 10^{2} \mathrm{~Pa} \cdot \mathrm{s}$ was determined (see above).

The high values of the zero shear viscosity imply that the mass transfer process evident from the overall decrease in the filament volume is limited by solvent diffusion from the mucilage [16, 36]. Using standard terminology for transport phenomena, this equates to large values of the mass transfer Biot number $B i=$ $h_{m c} R \alpha / D \gg 1$, where $h_{m c}$ (with units of $\left[m \cdot s^{-1}\right]$ ) is the convective mass transfer coefficient, $R$ is a characteristic length scale (the filament radius), $\alpha \approx 10^{-3}$ is the solvent partition coefficient (estimated 
from the partial densities of the solid and liquid phases [16]) and $D$ is the effective mass diffusivity of the solvent [37] (water) through the polymer solution. The coupled effects of capillary thinning and mass transfer can then be incorporated in the large Biot number limit by a simple lumped parameter model, giving rise to a time-dependent fluid viscosity [32]

$$
\eta(t)=\eta_{0} \exp \left(2 P \int_{0}^{t} \frac{R_{0}}{R(t)} d t\right)
$$

with the parameter $P=t_{v c} / t_{m}$, where $t_{v c}$ is the viscocapillary time and $t_{m}$ is the characteristic time scale for mass transfer. Assuming that solvent transport is diffusion-limited, this time scale is $t_{m}=R_{0}^{2} / D$ [33]. Consequently, $P$ is typically small: $P \approx 0.004$ with values $t_{v c}=0.4 s$ and $t_{m} \sim O\left(10^{2} s\right)$.

The thinning and breakup patterns of the viscoelastic mucilage and the PDMS silicone oil are markedly different. Whereas the liquid filaments of silicone oil undergo classical capillary thinning (and eventually break up) as expected for Newtonian liquids [38], the plant mucilage shows viscoelastic fluid behavior only during the initial phase $(t \lesssim 0.5 s)$ but then forms a stationary fiber for $t \gg t_{v c}$. In between those two regimes $\left(10^{-1} s \lesssim t \lesssim 10^{1} s\right)$, capillary thinning competes with mass transfer (inducing solvent loss and an overall increase in viscosity), and with extensional strain-stiffening. For the experiment shown in Fig. 5 with length scales of the order 1-10 $\mu \mathrm{m}$, the transition from viscocapillary thinning to the more complex behavior involving drying occurs around $t \approx 0.5 \mathrm{~s}$ after the initial stretching deformation.

The best parameter to characterize the biopolymer dynamics in the mucilage is the fluid relaxation time $\lambda_{e}$; its value can be obtained by fitting the initial portion of the extensional rheology data to a viscoelastic fluid model $[17,39]$ based on the temporal evolution of the minimum radius following

$$
\frac{R_{m}(t)-R_{\text {stat }}}{R_{0}}=\left(\frac{\eta_{0} R_{0}}{2 \lambda_{e} \sigma}\right)^{1 / 3} \exp \left(-\frac{t}{3 \lambda_{e}}\right) .
$$

The resulting value of $\lambda_{e}=0.33 \mathrm{~s}$ is similar to 
the characteristic time scale $\lambda_{c}=1 / \dot{\gamma}^{*} \approx 0.4 s$ obtained from the steady shear experiments described above; the minor discrepancy is due to non-idealities associated with minor deviations from the shape of a cylindrical liquid bridge, inherent to in-situ experiments [40] (see Fig. 5(a) at $t=0.00 \mathrm{~s}$ ).

In these experiments, the values of the Bond number $B o=\Delta \rho g R^{2} / \sigma$ are of the order $B o \approx 10^{-4}$, meaning that gravitational forces can be neglected as compared to capillary forces. Indeed, viscous, elastic and capillary forces exceed gravity by orders of magnitude and gravitational sagging of the liquid filament is irrelevant here.

$$
\text { [***MOVED TO DISCUSSION***] }
$$

\subsection{Fiber Formation and Capillary Instabilities at Large Deformations}

The material properties documented here have been described in the past empirically as 'spinnability' or 'stringiness' in the context of chemical engineering operations such as atomization or fiber spinning $[23,37]$. They become increasingly dominant in strong flows, involving higher extensional strains than those used above for capillary microrheometry. In Fig. 6 we show images for mucilage drops after they have been stretched rapidly to a larger pre-strain $\ln \left(L_{f} / L_{0}\right) \approx 2.6$ (stage translation time $80 \mathrm{~ms}$ ). Fig. 6a shows the onset of a Plateau-Rayleigh instability [21] leading to beads-on-a-string morphologies similar to those observed with dilute polymer solutions [43-46]. In addition to capillary thinning, bead formation here is not only influenced by strain-stiffening in the thin liquid ligaments [26] connecting the beads, but also by enhanced mass transfer of the solvent out of the liquid thread as it gets stretched [16, 32]. Ultimately, this combination results in a 'frozen' surface wave on the liquid thread [47]: the thin connecting ligaments solidify, and highly aligned, birefringent structures such as those seen in Fig. 6c are preserved indefinitely. Despite the small system size, the capillary pressure is insufficient to drive relaxation of the individual beads back to a near-spherical shape. In contrast to spherical beads of a Newtonian fluid on a wire [48], where the 
capillary pressure dominates viscous and elastic forces, the beads observed here usually retain an elongated shape, affected only by some additional decrease in total volume at long times.

In Fig. 7 we demonstrate how similar morphologies develop on an existing polymer fiber exposed to an atmosphere saturated with water vapor. First, a mucilage drop is elongated in an intermediate humidity environment ( $\varphi=0.4$, left-most images). Irregular breakup is observed: there is no distinguishable value of the most unstable wavelength that would dominate the breakup process. In terms of the classical filament breakup theories, this corresponds to a broad and flat dispersion curve typical for very viscous systems with Ohnesorge numbers $O h \gg 1$ [22]. We then rapidly quench the atmosphere to saturation $(\varphi \rightarrow 1)$ and thereby force condensation of water from the atmosphere on the thread surface. Within tens of milliseconds, this induces bead formation with a much shorter wavelength. These droplets formed under high humidity conditions establish an actual beads-on-a-fiber morphology [49] in the original meaning of the term, with a thin liquid coating on a solidified fiber, where in our case the solid is the previously formed, strain-stiffened viscoelastic thread. The nearly spherical shape of the beads in this case indicates that capillary forces dominate over the fluid viscosity, since the water has just condensed and therefore only contains minute amounts of solvated polymer. This is in contrast to the elongated beads described above (Fig. 6). The morphologies seen here are rather similar to the original 'beads-on-a-string' mentioned by C. V. Boys in his 1890 lectures [50]. Originally, the term had referred to a solid thread coated with an annular fluid film undergoing a Plateau-Rayleigh instability [21, 51, 52]. This problem was later analyzed by Goren [49], who investigated the instability of an annular coating of liquid on a solid thread (or, inversely, on the inside of a wetted capillary tube).

In summary, the coupled viscoelastic and mass transfer phenomena described herein make it difficult to break up a mucilage thread under the action of capillary thinning alone. At short times $t \sim \lambda$, and moderate Hencky strains, the initial viscocapillary flow leads to the familiar, smoothly-necked 'hourglass' shape and 
the initial phase of thinning is reminiscent of many other viscoelastic fluids [19]. However, as the radius decreases, extensional strain-stiffening, coupled with the enhanced solvent mass transfer associated with the large increase in the surface area/volume ratio eventually suppresses the capillary thinning flow, yielding a stationary, solid polysaccharide thread. Under slow stretching deformations, micrometer-thin fibers can be 'spun' from these threads as we show in Fig. 8a.

Paradoxically, the presence of non-solidified fluid beads on an otherwise dried thread further enhances the efficiency of Drosera mucilage as a bioadhesive. Filaments retaining some fluid beads can easily be further extended by stretching out the thread to form daughter beads. The dry segments between the beads of a stretched filament provide molecular orientation [53] (as observed by the birefringence of the dried threads, see Fig. 6) and tensile strength to the spun biopolymer; however, the beads themselves may remain liquid-like for extended times. This may be demonstrated by the simple experiment depicted in Fig. 8b: the first frame shows a single fluid bead that has been formed on a filament. Upon further stretching this fluid bead separates into two daughter droplets, enhancing the overall extensibility of the thread before breakage. As we discuss further below, this combination of fluidity and extensibility is strongly reminiscent of the 'windlass' mechanism employed by ecrillibate spiders in their capture silk [13, 54, 55].

When these solidified threads eventually break upon further elongation, it is due to failure within the rigid connecting ligaments (Fig. 8b), or due to adhesive failure at the surface of the substrate (Fig. 8c). Whereas strongly strain-stiffening fluids may be stabilized against necking instabilities upon stretching, they are prone to cohesive or adhesive instabilities near the solid boundary [56]. Interestingly, similar dynamic failure phenomena have been discussed previously in the context of industrial, pressure-sensitive adhesives [57]. Indeed, we never managed to break a thread of the mucilage capture fluid by capillary breakup alone: solidification occurs too rapidly, and extensional strain-stiffening is too strong for the fluid to break up in the manner shown above by the reference silicone oil (Fig. 5). Even in the case of high humidity, thread 
breakup is impossible to achieve by capillary thinning alone: the combined effects of polymer orientation in the extended ligaments between the beads are sufficient to resist the capillary forces and suppress filament breakup, leading to formation of a beads-on-a-string morphology [26].

\section{DISCUSSION AND CONCLUSIONS}

The capture mechanism of the sundew plant is based on a combination of the effects of extensional viscoelasticity, capillary thinning and a liquid-to-solid transition driven by solvent mass transfer. Four natural time scales are important in the process: the polymer relaxation time $\lambda$, the viscocapillary time $t_{v c}$, a characteristic time for mass transfer $t_{m}$, and the Rayleigh time $t_{r}$. Here, the system size and the rate at which the beads develop are in a range that mass transfer can dominate and the liquid thread solidifies even before the onset of sustained exponential thinning of the cylindrical ligaments connecting the beads. The large initial shear viscosities of the mucilage drops are necessary to sufficiently slow down the capillary thinning flow and allow solvent evaporation to dominate the dynamics (otherwise the very thin liquid threads would break up too rapidly). From this perspective, the sundew plant has 'optimized' the rheology of its mucilage to provide an efficient trapping mechanism at small length scales.

[***MOVED HERE FROM RESULTS... The small volumes of the mucilage along with its function as a biological adhesive only make this an interesting application of capillary microrheometry, but they also suggest an important role for extensional flows when prey comes into contact with the fluid, since any fluid motion is likely to involve rapid stretching deformation of the droplet. For flows of dilute polymer solutions, viscoelasticity becomes increasingly dominant as the scales get smaller, for example in microfluidic contraction flows [41, 42]. For free surface flows at small length scales, the additional effects of capillarity and mass transfer must be taken into account in the form of the additional characteristic timescales $t_{v c}$ and $t_{m}$. The ratio of the polymer relaxation time and the viscocapillary time defines a dimensionless group, 
the elastocapillary number $E c=\lambda / t_{v c}=\lambda \sigma / \eta_{0} R_{0}$. The magnitude of this parameter is important in filament thinning processes and this ratio can be viewed as a modified form of the well-known Deborah number $D e=\lambda \dot{\epsilon}$, where the extension rate $\dot{\epsilon}$ has been replaced with the inverse of the viscocapillary time $t_{v c}^{-1}$. We find typical values in the range of $E c \approx 0.1$ for Drosera mucilage. This can be directly compared to the value of the elastocapillary number $E c$ calculated for the mucilage of the much larger pitcher plant (Nepenthes): using previously published data to evaluate the viscocapillary and polymer relaxation times [5], we find typical values $E c \approx 0.5-1$ for the pitcher plant (notice that in the case of the pitcher plant the available fluid volumes were larger, therefore macroscopic capillary breakup experiments could be performed by Gaume and Forterre [5]). For the capture fluids of both plants, smooth elastocapillary thinning flows with characteristic time scales of the order of seconds can develop. The extensional rheology data strongly suggest that in both species extensional strain-stiffening plays an important role in the capturing mechanism.

There is, however, a key difference: Drosera is capable of capturing insects with very small fluid volumes in the sub-nanoliter range and much of the surface of the prey remains unwetted. On the other hand, Nepenthes relies on a mechanism in which prey remains trapped in a relatively large volume of the viscoelastic capture liquid with comparatively low shear viscosity but high transient extensional viscosities; indeed it was reported that pitcher plant mucilage even retained its capturing efficiency upon dilution with water [5]. In contrast, a capturing mechanism relying exclusively on the enhanced extensional viscosity of a polymer solution would be ineffective for Drosera: the magnitude of the extensional stresses generated by stretching the tiny, sub-nanoliter mucilage droplets are not sufficient to capture insects. Mucilage present in such small volumes appears to be effective as a capture fluid only if the extended threads can undergo a liquid-to-solid transition by drying. The key property here is 'spinnability' $[33,37,58,59]$, a complex quantity that has been used empirically in fiber processing and is related to several physical pa- 
rameters including extensional viscosity, zero shear viscosity, mass transfer parameters, system length scale and capillarity. Most importantly, the dimensionless ratio between the viscocapillary and the mass transfer time scales $P=t_{v c} / t_{m}$ should be in a range that allows drying of the strain-stiffened liquid threads before breakup occurs (here: $P \gtrsim 10^{-3}$ at $E c \approx 0.1$ ). For Drosera, this implies that the small system size must be compensated for with a high shear viscosity, since capillary break up would otherwise occur too rapidly. Indeed, the measured zero shear viscosity (Fig. 3) is sufficiently high that necking flows remain relatively slow even for small liquid bridges with radii of less than $10 \mu \mathrm{m}$, with viscocapillary time scales in a range where the drying process dominates and ultimately 'freezes' the extended mucilage threads. In terms of dimensionless groups, this implies that the ratio $P$ should be neither very small nor very large, since otherwise either capillary breakup of the thinning thread would pre-empt solidification of the extended liquid bridge $(P \ll 1)$, or the mucilage would dry too quickly for it to be stretched into an extended filament $(P \gg 1)$. Unlike the elastocapillary number $E c$, which appears to be in a similar range for both plants, the parameter $P$ accordingly varies by orders of magnitude between the mucilage fluids of Drosera $\left(P \gtrsim 10^{-3}\right)$ and Nepenthes $\left(P \lesssim 10^{-5}\right)$ due to the different viscocapillary time scales. ... MOVED HERE FROM RESULTS***]

For polymer solutions, the formation of beads-on-a-string structures is determined by a delicate balance of the forces of capillarity, viscosity, elasticity and inertia [46], with the relative magnitudes of each of these effects being neither very small nor large. These morpologies have been studied in the context of materials processing, in particular for electrospinning applications [60]. Due to the strong influence of mass transport, a full analysis of the free surface dynamics observed here remains a considerable challenge. Recent efforts combining extensional flows of dilute polymer solutions with strong solvent mass transport combine numerical modeling with experimentally determined rheological material functions [33]; this approach is also expected to provide additional insight into biological fiber formation processes. A similar droplet and 
filament 'windlass' mechanism is used in the capture spirals of some orb-weaving spiders [54] where balls of a loose core fiber are stored within aqueous glue drops and are unwound under tension. The images shown in Fig. 6 indicate that under humid conditions Drosera mucilage threads are similar to those structures and consist of a highly-birefringent and flow-aligned solid core fiber surrounded by a lower viscosity liquid coating. Ultimately, this implies that the microstructure and the local viscosity are radially inhomogeneous in the thread. For thread configurations with a large surface-to-volume ratio, fluid inhomogeneity becomes important. A low-viscosity fluid layer covering a much more viscous or solidified polymeric core also helps explain the short wavelengths of the surface instabilities shown in Fig. 7. Beads formed by a low viscosity coating layer are consistent with the liquid annulus picture, wherein the observed instability is controlled by the viscosity and the thickness of the annular coating $[49,50]$. If the highly extended threads were composed of a homogeneous, viscoelastic fluid, the corresponding values of the Ohnesorge and Deborah numbers are far outside the regime wherein beads-on-a-string structures are expected [66] A low viscosity coating can also locally plasticize and partially dissolve biopolymer from the surface of the thread as has been described for spider silk [54]; the hygroscopic nature of the drosera mucilage [2] appears to play an important role for this. Partial dissolution of polymer out of the central thread into a coating of condensed water from the atmosphere would ultimately lead to an increase of the viscosity of such a coating layer. Finally, similar liquid bridges can be observed in unexpected places when high molecular weight biopolymer liquids are subjected to strong flows. For example, they have been visualized by SEM for the mucilage connecting cells of the diatom Melosira varians under turbulent flow conditions [61]. In that case the diatoms act as $\mu \mathrm{m}$-sized end plates stretching the connecting liquid bridges to the familiar hourglass shape in a strong external flow field.

The rather simple chemical composition of the plant mucilage studied here is in stark contrast to the compositional and structural complexity of many biological fibers [62], silks [54, 
55, 63], or mucins [64] and slimes [65], yet it provides an effective capturing system that is adapted to match the range of the forces exerted on the liquid threads by a trapped insect.

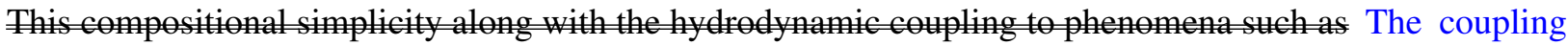
of capillary thinning and mass transfer phenomena applied at appropriate length and time scales might serve as an inspiration for future research into biological free surface flows of complex fluids. It would also be interesting to measure the mechanical properties of individual thin dried polysaccharide fibers and compare them to high-strength biomaterials with improved functionality.

\section{Acknowledgements}

This work was partially supported by NASA as part of the Microgravity Science Glovebox program (SHERE project). PE acknowledges Irene Lichtscheidl and Wolfram Adlassnig (Cell Imaging and Ultrastructure Research, University of Vienna) for bringing several interesting publications on carnivorous plants to our attention. CC acknowledges financial support from the ERC starting grant 203043 NANOFIB. GHM would like to thank Christophe Clanet (École Polytechnique, France) for initial discussions on the topic of Drosera. 
[1] C. Darwin, Insectivorous plants, John Murray, London, 1875.

[2] F. G. Clancy and M. D. Coffey, Can. J. Bot. - Rev. Can. Bot., 1977, 55, 480-488; S. E. Williams and B. G. Pickard, Planta, 1972, 103, 193-221; K. Rost and R. Schauer, Phytochemistry, 1977, 16, 13651368; D. C. Gowda, G. Reuter and R. Schauer, Phytochemistry, 1983, 21, 2297-2300; D. C. Gowda, G. Reuter and R. Schauer, Carbohydr. Res., 1983, 113, 113-124; G. O. Aspinali and V. Puvanesarajah, Carbohydr. Res., 1984, 131, 53-60.

[3] Y. Forterre, J. M. Skotheim, J. Dumais and L. Mahadevan, Nature, 2005, 433, 421-425.

[4] D. P. Holmes and A. J. Crosby, Adv. Mat., 2007, 19, 3589-3593.

[5] L. Gaume and Y. Forterre, PLoS ONE, 2007, 2, e1185.

[6] M. L. Gardel, M. T. Valentine, J. C. Crocker, A. R. Bausch and D. A. Weitz, Phys. Rev. Lett., 2003, 91, 158302.

[7] T. A. Waigh, Rep. Prog. Phys., 2005, 68, 685-742.

[8] A. R. Bausch, F. Ziemann, A. A. Boulbitch, K. Jacobson and E. Sackmann, Biophys. J., 2003, 75, $2038-2049$.

[9] E. M. Furst, Curr. Opin. Colloid Interface Sci., 2005, 10, 79-86.

[10] M. J. Solomon and Q. Lu, Curr. Opin. Colloid Interface Sci., 2001, 6, 430-437.

[11] A. J. Levine and T. C. Lubensky, Phys. Rev. Lett., 2000, 85, 1774-1777.

[12] S. Orso, U. G. K. Wegst, C. Eberl and E. Arzt, Adv. Mater., 2006, 18, 874-877.

[13] V. Sahni, T. A. Blackledge and A. Dhinojwala, Nature Comm., 2010, 1, 19.

[14] H. M. Wyss, T. Franke, E. Mele and D. A. Weitz, Soft Matter, 2010, 6, 4550-4555.

[15] M. Guo and H. M. Wyss, Macromol. Mat. Eng., 2011, 296, 223-229. 
[16] N. Kojic, J. Bico, C. Clasen and G. H. McKinley, J. Exp. Biol., 2006, 209, 4355-4362; N. Kojic, M. Kojic, S. Gudlavalletti and G. H. McKinley, Biomacromolecules, 2004, 5, 1698-1707.

[17] V. M. Entov and E. J. Hinch, J. Non-Newtonian Fluid Mech., 1997, 72, 31-53.

[18] S. L. Anna and G. H. McKinley, J. Rheol., 2001, 45, 115-138.

[19] G. H. McKinley, Rheology Reviews, The British Society of Rheology, Aberystwyth UK, 2005, pp. $1-48$.

[20] M. Stelter, G. Brenn, A. L. Yarin, R. P. Singh and F. Durst, J. Rheol., 2002, 46, 507-527.

[21] P. G. de Gennes, F. Brochard-Wyart and D. Quéré, Capillarity and Wetting Phenomena, Springer, New York, 2004.

[22] J. Eggers, Rev. Mod. Phys., 1999, 69, 865-929.

[23] L. E. Rodd, T. P. Scott, J. J. Cooper-White and G. H. McKinley, Appl. Rheol., 2005, 15, 12-27.

[24] L. Campo-Deano and C. Clasen, J. Non-Newtonian Fluid Mech., 2010, 165, 1688.

[25] W. von Ohnesorge, Z. Angew. Math. Mech., 1936, 16, 355; M. J. McCarthy and N. A. Malloy, Chem. Eng. J., 1974, 7, 1-20; S. P. Lin and R. D. Reitz, Ann. Rev. Fluid Mech., 1998, 30, 85-105.

[26] B. P. Bhat, S. Appathurai, M. T. Harris, M. Pasquali, G. H. McKinley and O. A. Basaran, Nature Physics, 2010, 6, 625-631.

[27] P. E. Arratia, J. P. Gollub and D. J. Durian, Phys. Rev. E, 2008, 77, 036309.

[28] L. A. Slobozhanin and J. M. Perales, Phys. Fluids, 1993, 5, 1305-1314.

[29] G. J. C. Braithwaite and G. H. McKinley, Appl. Rheol., 1999, 9, 27-33; C. Clasen, B. P. Gearing and G. H. McKinley, J. Rheol., 2006, 50, 883-905; S. Gudlavalleti, B. P. Gearing and L. Anand, Exp. Mech., 2005, 45, 412-419; C. Clasen and G. H. McKinley, J. Non-Newtonian Fluid Mech., 2004, 124, $1-10$.

[30] K. Yasuda, R. C. Armstrong and R. E. Cohen, Rheol. Acta., 1981, 20, 163-178. 
[31] D. T. Papageorgiou, Phys. Fluids, 1995, 7, 1529-1544.

[32] A. Tripathi, P. Whittingstall and G. H. McKinley, Rheol. Acta, 2000, 39, 321-337.

[33] J. Crest, J. C. Nave, S. Pabba, R. W. Cohn and G. H. McKinley, J. Non-Newtonian Fluid Mech., 2010, in press.

[34] E. Miller, C. C. and J. P. Rothstein, Rheol. Acta, 2009, 48, 625-639.

[35] R. G. Larson, The Structure and Rheology of Complex Fluids, Oxford University Press, Oxford, UK, 1999.

[36] G. A. Truskey, F. Yuan and D. F. Katz, Transport Phenomena in Biological Systems, 2nd Ed., Pearson, New York, 2010.

[37] A. Ziabicki, Fundamentals of Fibre Formation. The Science of Fibre Spinning and Drawing, Wiley, London, 1976.

[38] G. H. McKinley and A. Tripathi, J. Rheol., 2000, 44, 653-669.

[39] C. Clasen, J. Eggers, M. A. Fontelos and G. H. McKinley, J. Fluid Mech., 2006, 556, 283-308.

[40] C. Wagner, Y. Amarouchene, D. Bonn and J. Eggers, Phys. Rev. Lett., 2005, 95, 164504.

[41] T. M. Squires and S. R. Quake, Rev. Mod. Phys., 2005, 77, 977-1026.

[42] M. S. N. Oliveira, L. E. Rodd, G. H. McKinley and M. A. Alves, Microfluid Nanofluid, 2008, 5, 809-826.

[43] M. S. N. Oliveira, R. Yeh and G. H. McKinley, J. Non-Newtonian Fluid Mech., 2006, 137, 137-148.

[44] J. Li and M. A. Fontelos, Physics of Fluids, 2003, 15, 922-937.

[45] B. Steinhaus, A. Q. Shen and R. Sureshkumar, Phys. Fluids, 2007, 19, 073103.

[46] P. Schümmer and K. H. Tebel, J. Non-Newtonian Fluid Mech., 1983, 12, 331-347.

[47] P. Erni, C. Cramer, I. Marti, E. J. Windhab and P. Fischer, Adv. Colloid Interface Sci., 2009, 150, $16-26$ 
[48] T. Gilet, D. Terwagne and N. Vandewalle, Appl. Phys. Lett., 2009, 95, 014106.

[49] S. L. Goren, J. Fluid Mech., 1961, 12, 309-319.

[50] C. V. Boys, Soap bubbles: their colors and forces which mold them, Dover Publications, New York, 1958.

[51] J. Plateau, Acad. Sci. Bruxelles Mém., 1849, 23, 5;.

[52] Lord Rayleigh, Proc. London Math. Soc., 1879, 10, 4-13.

[53] R. Sattler, A. Kityk and C. Wagner, Phys. Rev. E., 2007, 75, 051805; R. Sattler, C. Wagner and J. Eggers, Phys. Rev. Lett., 2008, 100, 051805; A. Glisovic, T. Vehoff, R. J. Davies and T. Salditt, Macromolecules, 2008, 41, 390-398.

[54] F. Vollrath and D. T. Edmonds, Nature, 1989, 340, 305-307.

[55] M. Heim, D. Keerl and T. Scheibel, Angew. Chem. Int. Ed., 2009, 48, 3584-3596.

[56] G. H. McKinley and T. Sridhar, Ann. Rev. Fluid. Mech., 2002, 34, 375-415.

[57] A. J. Crosby, K. R. Shull, H. Lakrout and C. Creton, J. Appl. Phys., 2000, 60, 5030-5042.

[58] T. Peltola, M. Jokinen, S. Veittola, H. Rahiala and A. Yli-Urpo, Biomaterials, 2001, 22, 589-598.

[59] C. J. S. Petrie, Elongational Flows, Pitman, London, UK, 1979.

[60] J. H. Yu, S. V. Fridrikh and G. C. Rutledge, Polymer, 2006, 47, 4789-4797.

[61] S. J. Clarson, M. Steinitz-Kannan, S. V. Patwardhan, R. Kannan, R. Hartig, L. Schloesser, D. W. Hamilton, J. K. A. Fusaro and R. Beltz, Silicon, 2009, 1, 79-90.

[62] M. Guthold, W. Liu, E. A. Sparks, L. M. Jawerth, L. Peng, M. Falvo, R. Superfine, R. R. Hantgan and S. T. Lord, Cell Biochem. Biophys., 2007, 49, 165-181.

[63] M. Moriya, F. Roschzttardtz, Y. Nakahara, H. Saito, Y. Masubuchi and T. Asakura, Biomacromolecules, 2009, 10, 929-935.

[64] J. P. Celli, B. S. Turner, N. H. Afdhal, R. Ewoldt, G. H. McKinley, R. Bansil and S. Erramilli, 
Biomacromolecules, 2006, 8, 1580-1586.

[65] D. S. Fudge, T. Winegard, R. H. Ewoldt, D. Beriault, L. Szewciw and G. H. McKinley, Integrat. Comparat. Biol., 2009, 49, 32-39.

[66] This is true even if smaller values of the shear viscosity due to shear thinning are taken into account: using a characteristic shear rate of the order $\dot{\gamma}=O\left(10^{3} s^{-1}\right)$ associated with the stretching motion of the thread, the shear viscosity and concomitantly the Ohnesorge number are decreased by an order of magnitude. 
Figures 


\section{Figures}

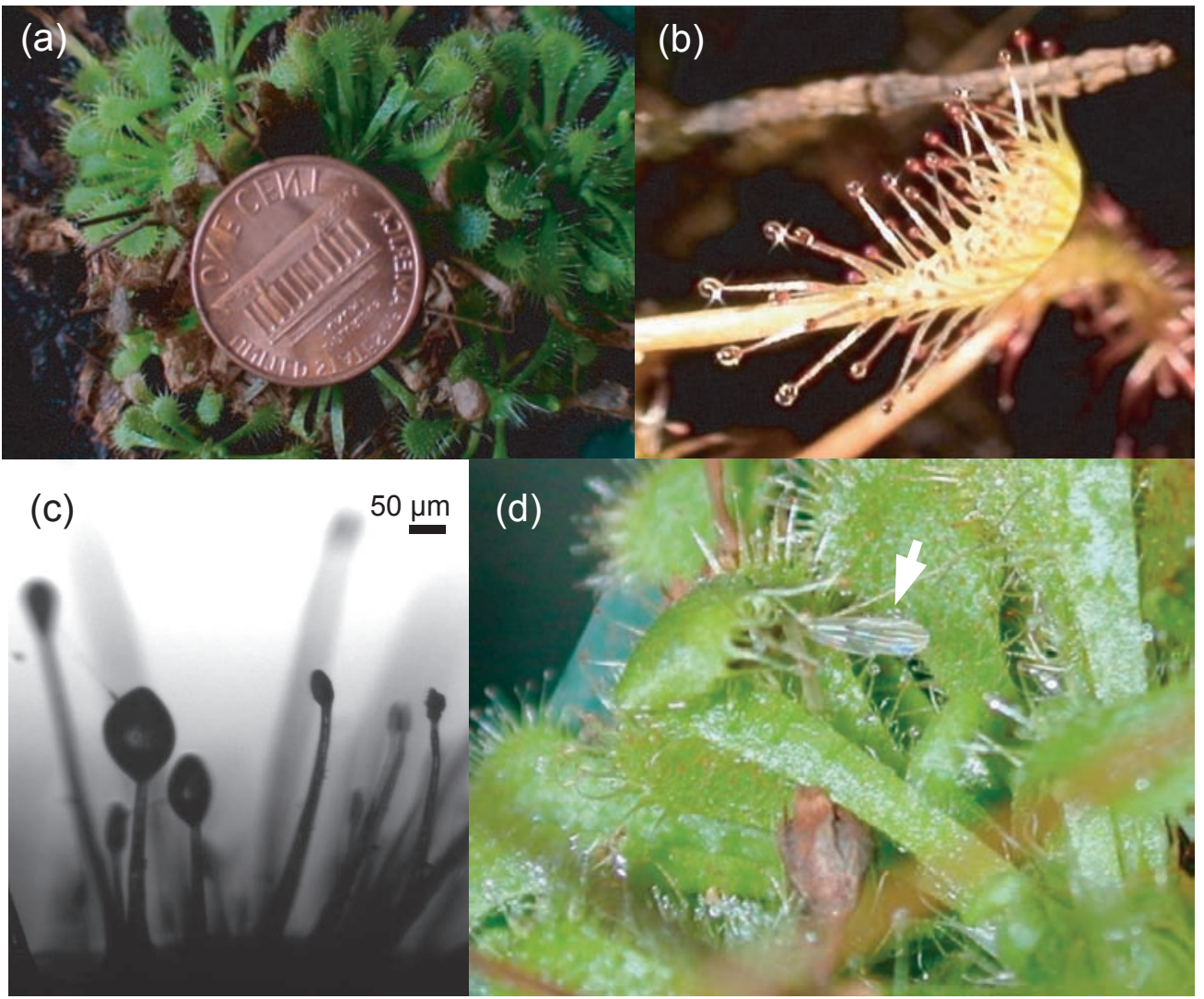

(e) $\left[\begin{array}{cc}\rightarrow 4)-\beta-D-G l c p A-(1 \rightarrow 2)-\alpha-D-M a n p-(1 \rightarrow 4)-\beta-D-G l c p A-(1 \rightarrow 2)-\alpha-D-M a n p-(1- \\ 3 & 3 \\ \uparrow & \uparrow \\ 1 & \alpha-D-\text { Galp } \\ \text { L-Araf } & \end{array}\right]_{n}$

$\left[\begin{array}{cc}\rightarrow 4)-\beta-D-G l c p A-(1 \rightarrow 2)-\alpha-D-M a n p-(1 \rightarrow 4)-\beta-D-G l c p A-(1 \rightarrow 2)-\alpha-D-M a n p-(1- \\ 3 & 3 \\ \uparrow & 1 \\ 1 & \alpha-D-G a l p \\ \alpha-D-X y l p & \end{array}\right]_{m}$

FIG. 1: The sundew plant shown at three different length scales and during the capturing process: (a) whole Drosera plant; leaves on the right hand side carry fluid drops; (b) single leaf with secreted mucilage drops; (c) individual mucilaginous glands as seen by brightfield light microscopy; (d) insect (indicated by the arrow) trapped in a rolled-up leaf; (e) composition of the linear main polysaccharide isolated from Drosera mucilage [2]. It is composed of a D-glucurono-D-mannan backbone with alternating glucuronic acid (GlcpA) and mannose (Manp) residues; standard notation for polysaccharide chemistry is used, with arrows indicating glycosidic bonds, three letter codes identifying the monosaccharide residues and ' $\mathrm{p}$ ' and ' $\mathrm{f}$ ' identifying pyranose or furanose rings. 
(a)

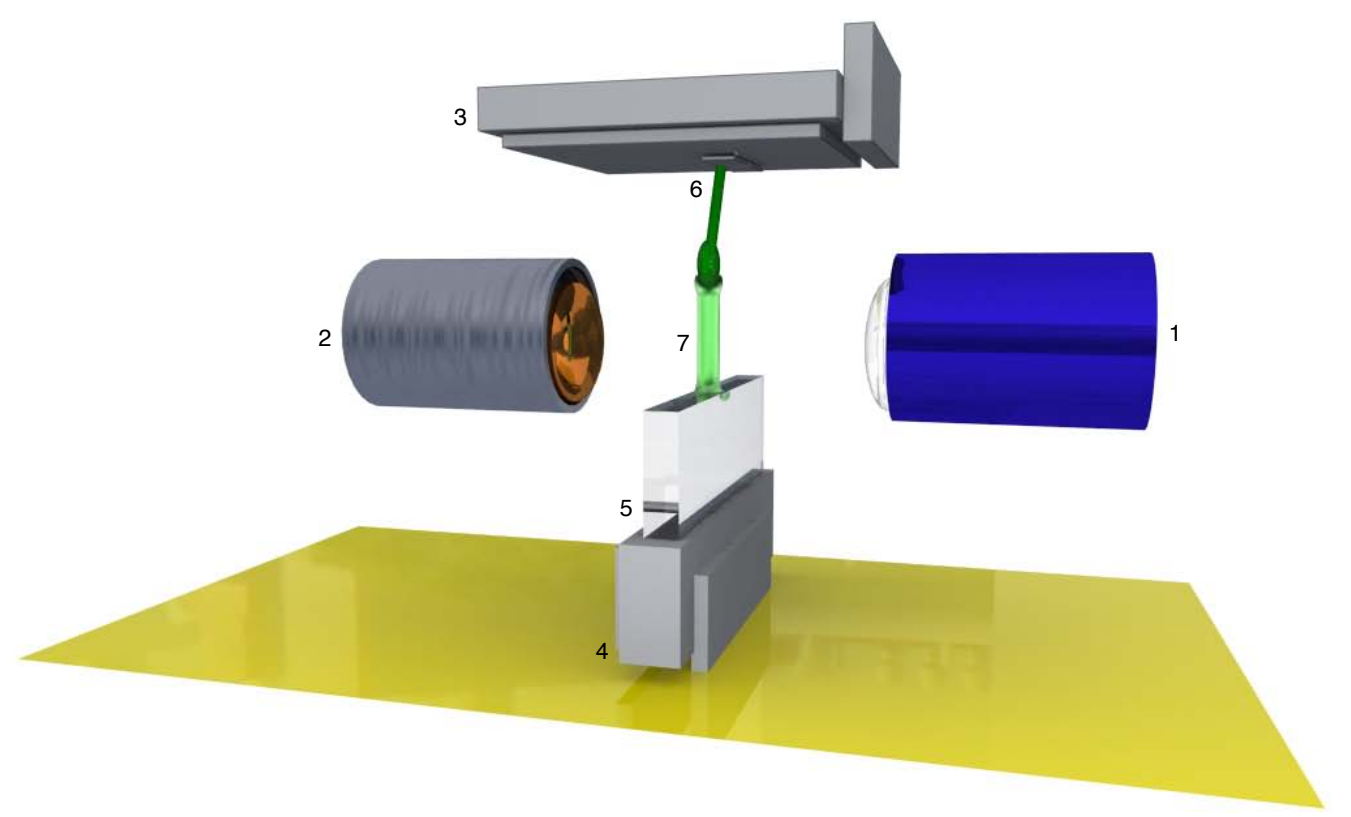

(b)
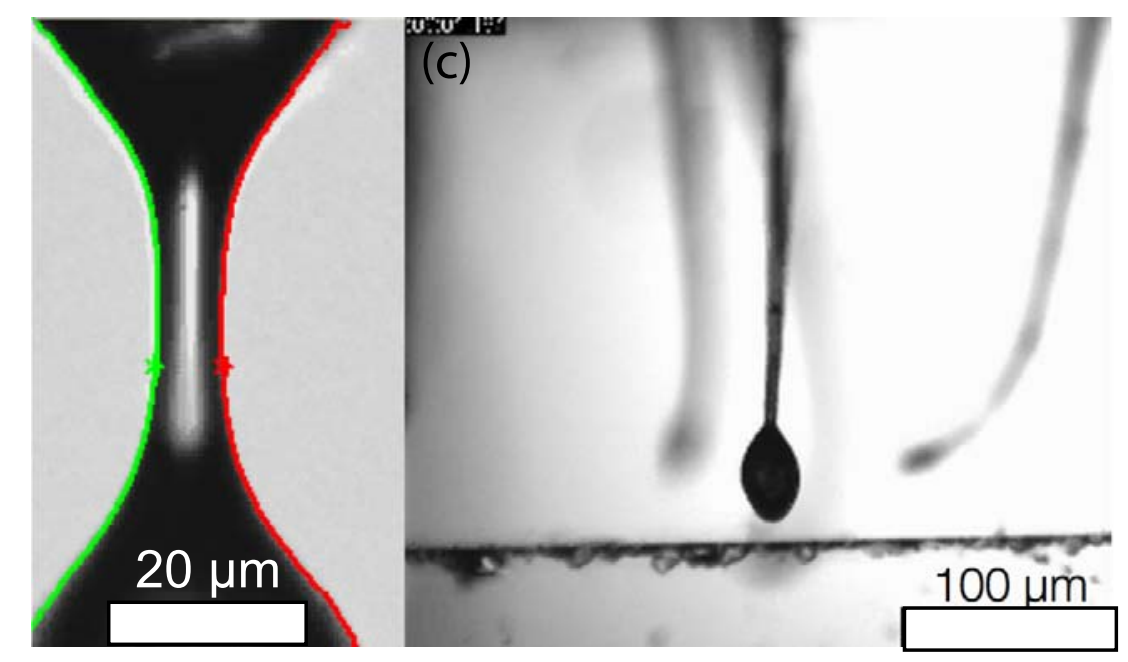

FIG. 2: Microscope-based capillary thinning rheometry; (a) experimental setup with microscope and translation stages; (1) microscope lens; (2) light source; (3) XYZ stage; (4) linear stage; (5) glass endplate; (6) sample holder with with single Drosera leaf or tentacle; (7) liquid bridge formed between plant tentacle and glass endplate Since in most cases the Bond number is small enough to neglect gravitational sagging, the liquid bridge may also be oriented horizontally. (b) Sample micrograph of a liquid bridge with the superimposed results of the edge detection procedure; (c) single plant tentacle holding a mucilage droplet approaches the side surface of microscope glass slide. 


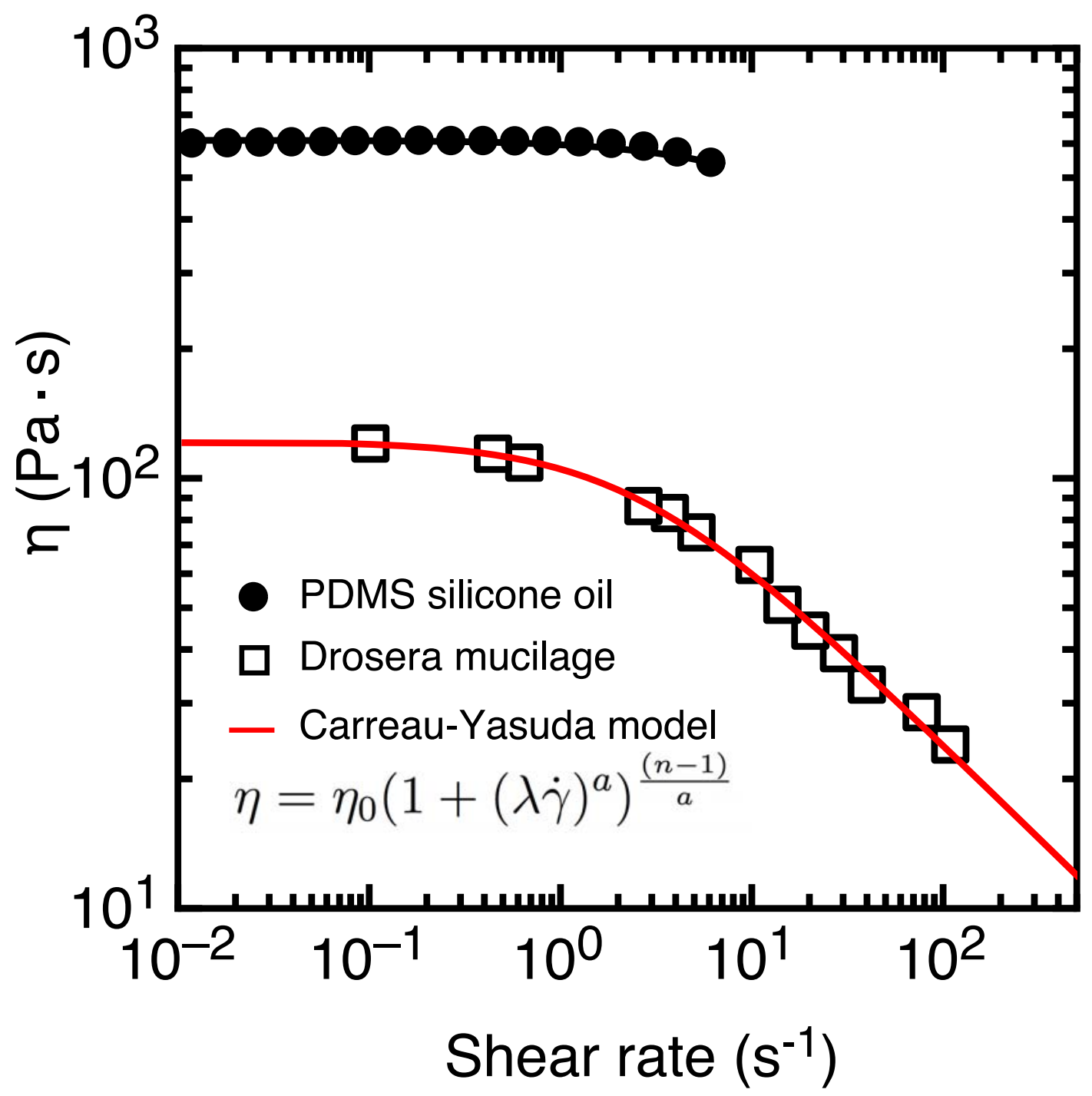

FIG. 3: Flow curve of Drosera mucilage measured in steady shear flow. Measurements are performed using a flexure-based microrheometer [29]. The Carreau-Yasuda model (solid lines) is used to describe the flow curve by nonlinear regression; parameters are the relaxation time $\lambda$, the zero shear viscosity $\eta_{0}$, the shear thinning exponent $n$, and an additional model parameter $a$ that controls the shear thinning transition (fitted values: $\left.\lambda=0.4 s ; \eta_{0}=122 P a \cdot s ; n=0.56 ; a=0.96\right)$. A PDMS silicone oil viscosity standard is included for comparison, with $\eta_{0}=605 \mathrm{~Pa} \cdot s$. 
(a)

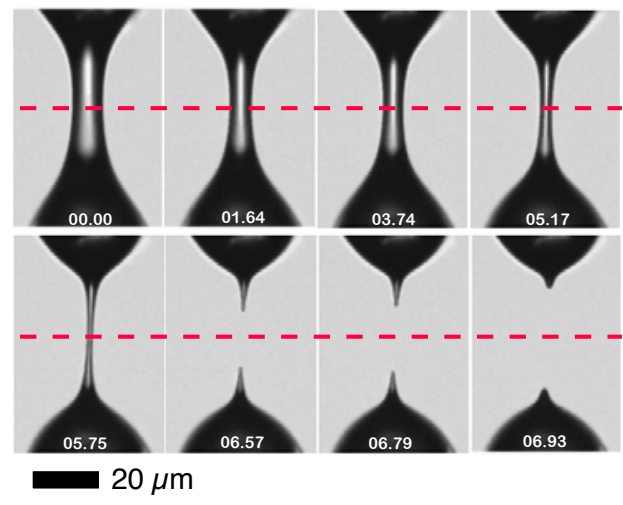

(b)

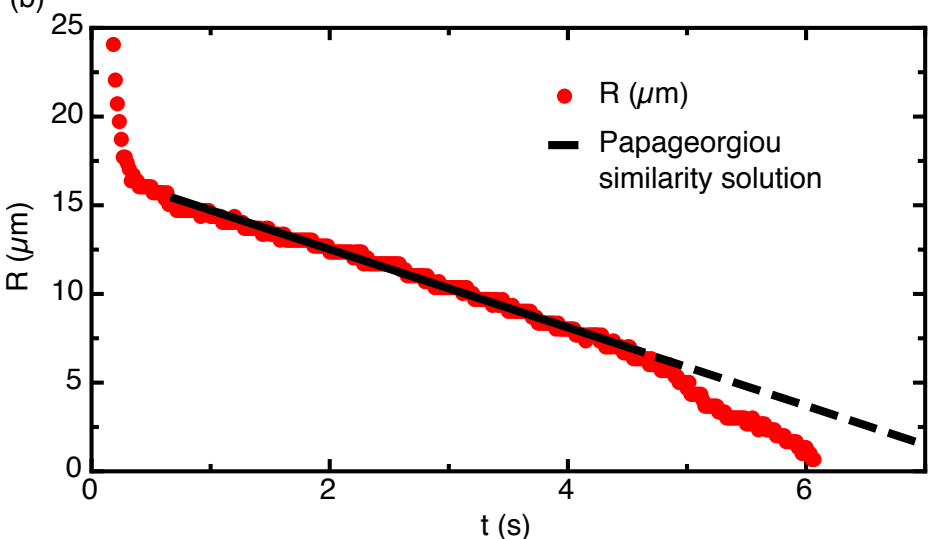

FIG. 4: Capillary breakup of a reference silicone oil and corresponding fit to the similarity solution for capillary thinning of a viscous Newtonian fluid thread [31]. Here the capillary thinning flow proceeds until the fluid filament breaks up; the extensional viscosity $\eta_{e}=3 \eta_{0}$ is constant with a value of three times the shear viscosity. Due to the high viscosity even the recoil of the cusped threads after capillary breakup takes several seconds to complete. Data from $t=0.75 \mathrm{~s}$ to $t=4.4 \mathrm{~s}$ were used for curve fitting. Time stamp for video images is indicated in seconds; $0.00 \mathrm{~s}$ is the time at which axial pre-stretching ceases.
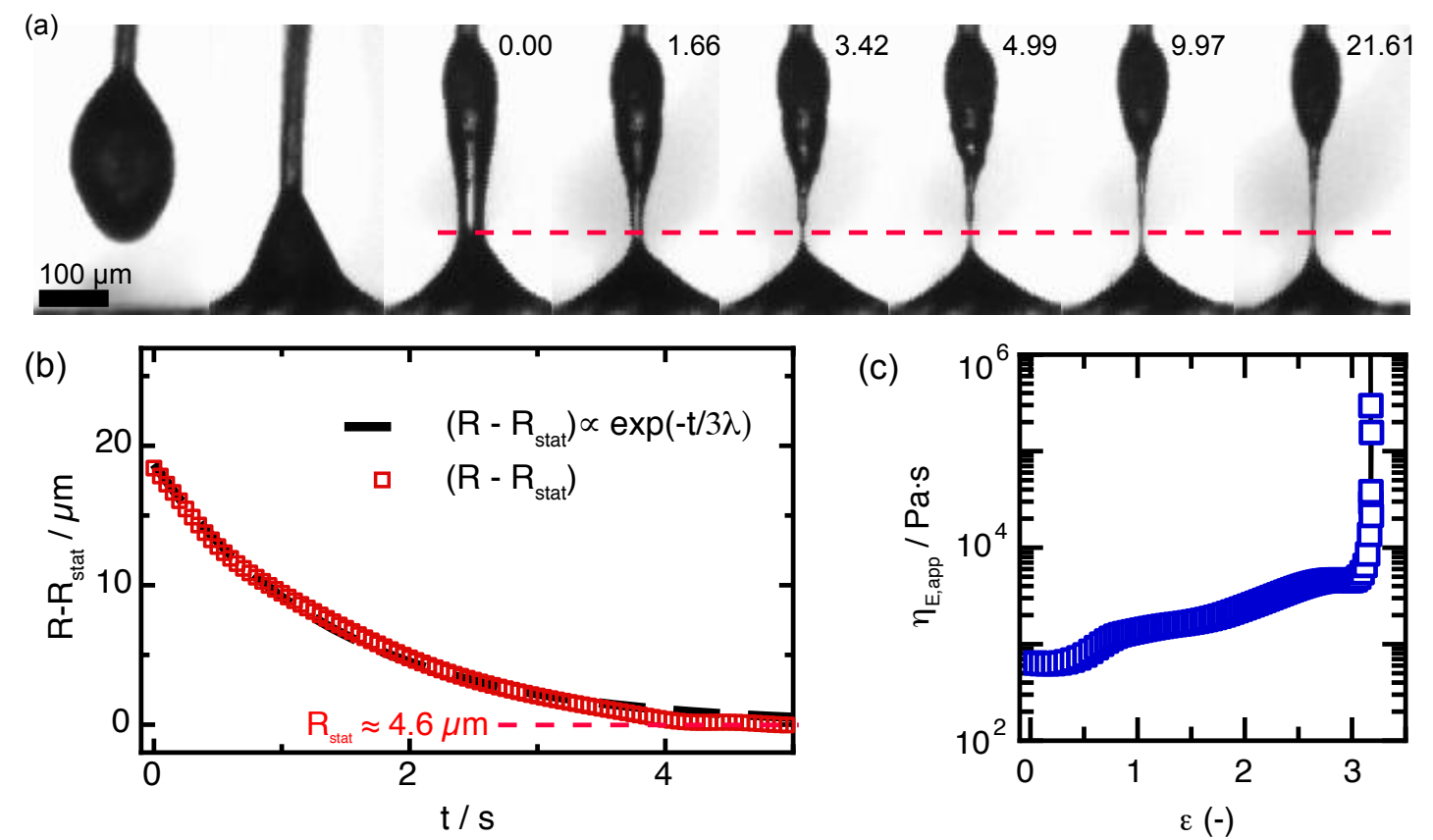

FIG. 5: (a) In-situ capillary thinning experiment with a mucilage drop on a single tentacle; time indicated with each frame is given in seconds; 0.00 s is the time at which axial stretching pre-ceases (the time for stretching is $120 \mathrm{~ms}$ ). The dashed line indicates the selected plane chosen to measure the radius, based on the minimum filament diameter $R_{\text {stat }}$ in the long time limit; relative humidity $\varphi=0.3$. (b) Evolution of the minimum radius $R_{m}(t)$ of the necking thread; the stationary radius $R_{\text {stat }}$ measured after thinning has halted is indicated on the right hand side. (c) Apparent extensional viscosity $\eta_{E, a p p}$ as a function of the accumulated Hencky strain $\epsilon$ in the material. 
(a)

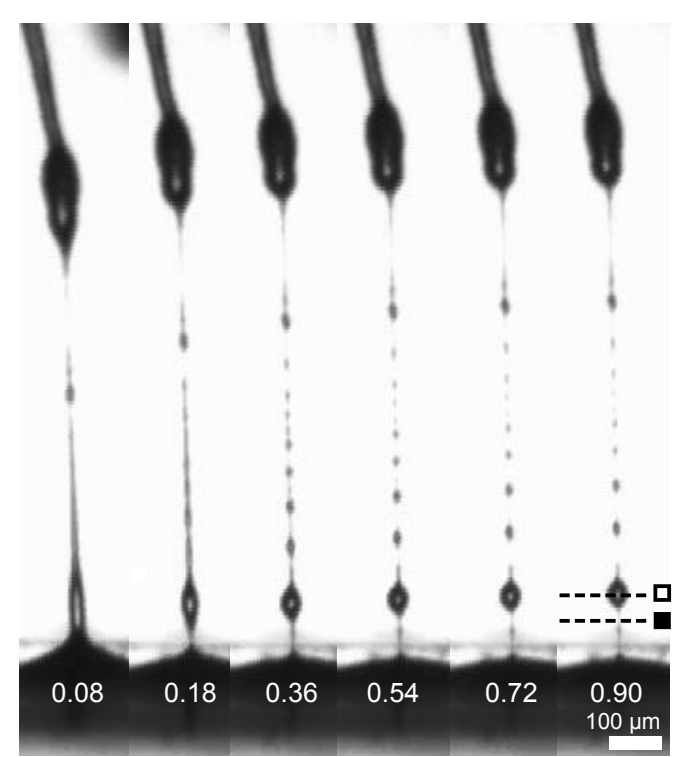

(b)

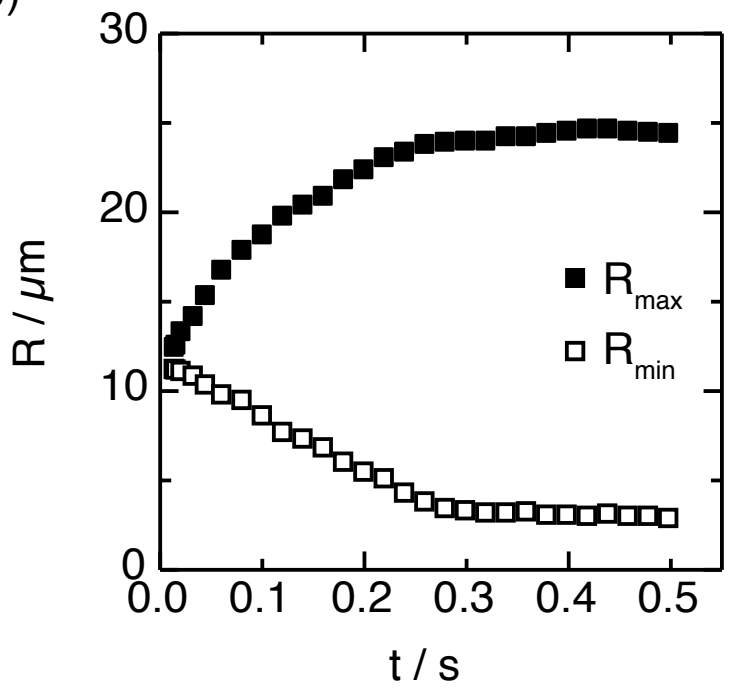

(c)

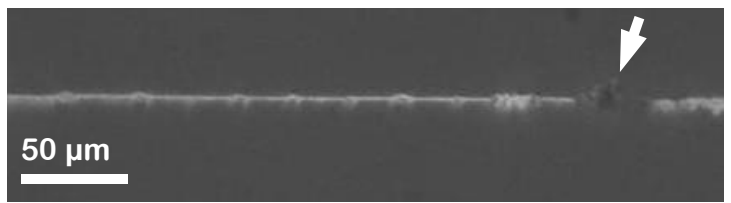

FIG. 6: Filament morphology for drops stretched rapidly to large strains: (a) Onset of Plateau-Rayleigh instability, giving rise to an evaporatively-stabilized beads-on-a-string morphology. Time is indicated in seconds. (b) Selected radii measured from video images of the experiment shown in (a). Despite the higher extensional pre-strains and, consequently, the smaller diameters as compared to the extensional rheometry experiments (Fig. 5) the filament resists capillary breakup; stationary values for the radii are reached after approximately $0.3 \mathrm{~s}$ and are retained indefinitely. Thinning is measured on the largest bead (see lines indicating the planes used to measure the thread radius). (c) Beads-on-a-string morphology pulled from Drosera mucilage as seen between crossed polarizers. The intensity of light transmitted provides a measure of flow-induced alignment in the dried mucilage thread. The arrow indicates a large wet and relaxed fluid which is not birefringent, connected by a highly aligned birefringent solid thread. 


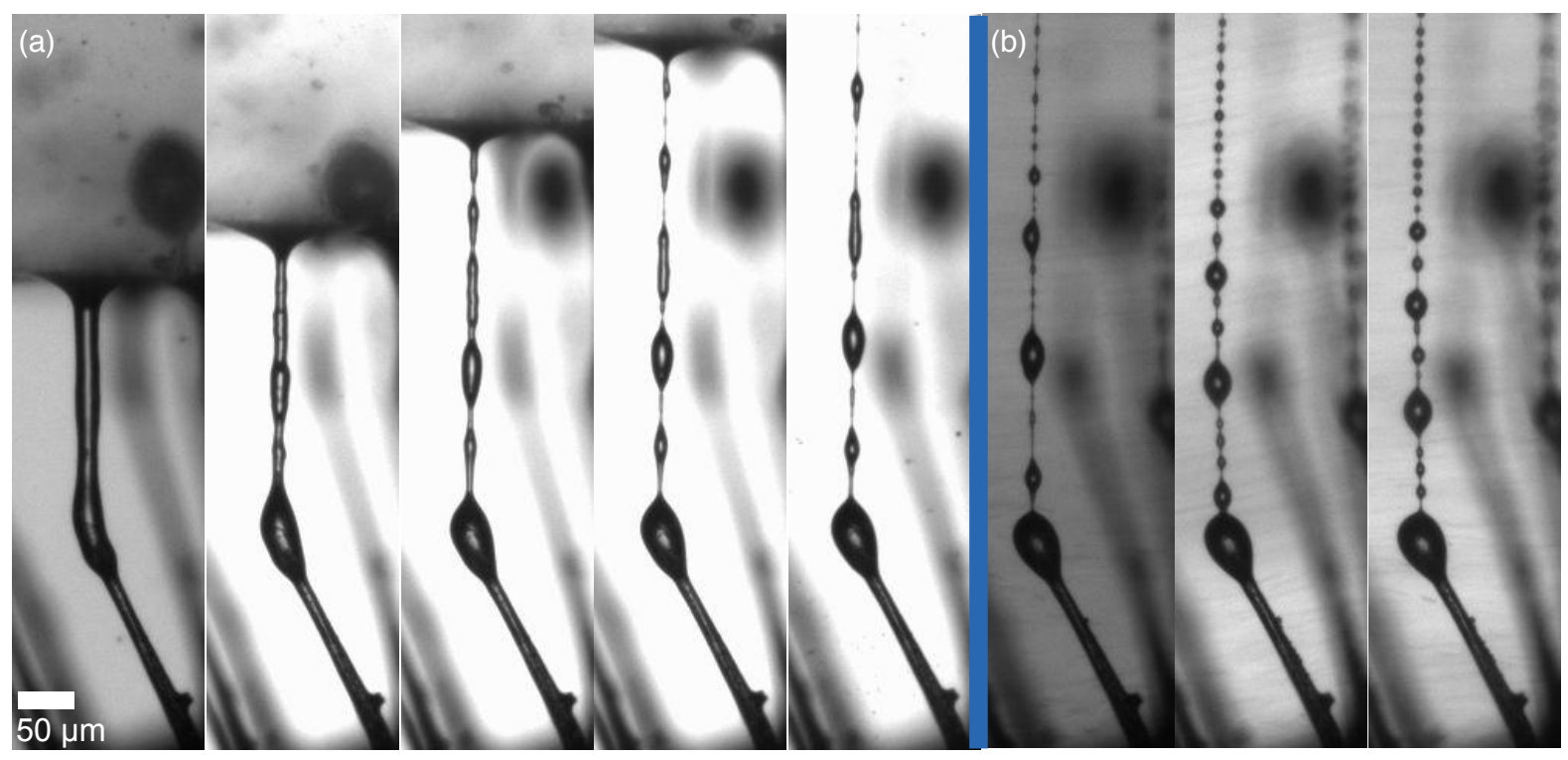

FIG. 7: (a) Filament stretched at intermediate humidity, developing irregular breakup expected for highly viscous threads; time step between images: $1 \mathrm{~s}$. (b) Same filament following a quench in relative humidity up to saturation; beads of nearly spherical shape form quickly from the condensed water on the thread. In this case, the original bead-to-bead distance corresponds to shorter and more regular wavelengths. As the beads grow from continued condensation, the Bond number increases and gravity leads to beads sliding downwards on the thread and sequentially coalescing with each other; time step between images: $150 \mathrm{~ms}$. 
(a)

$\circ$

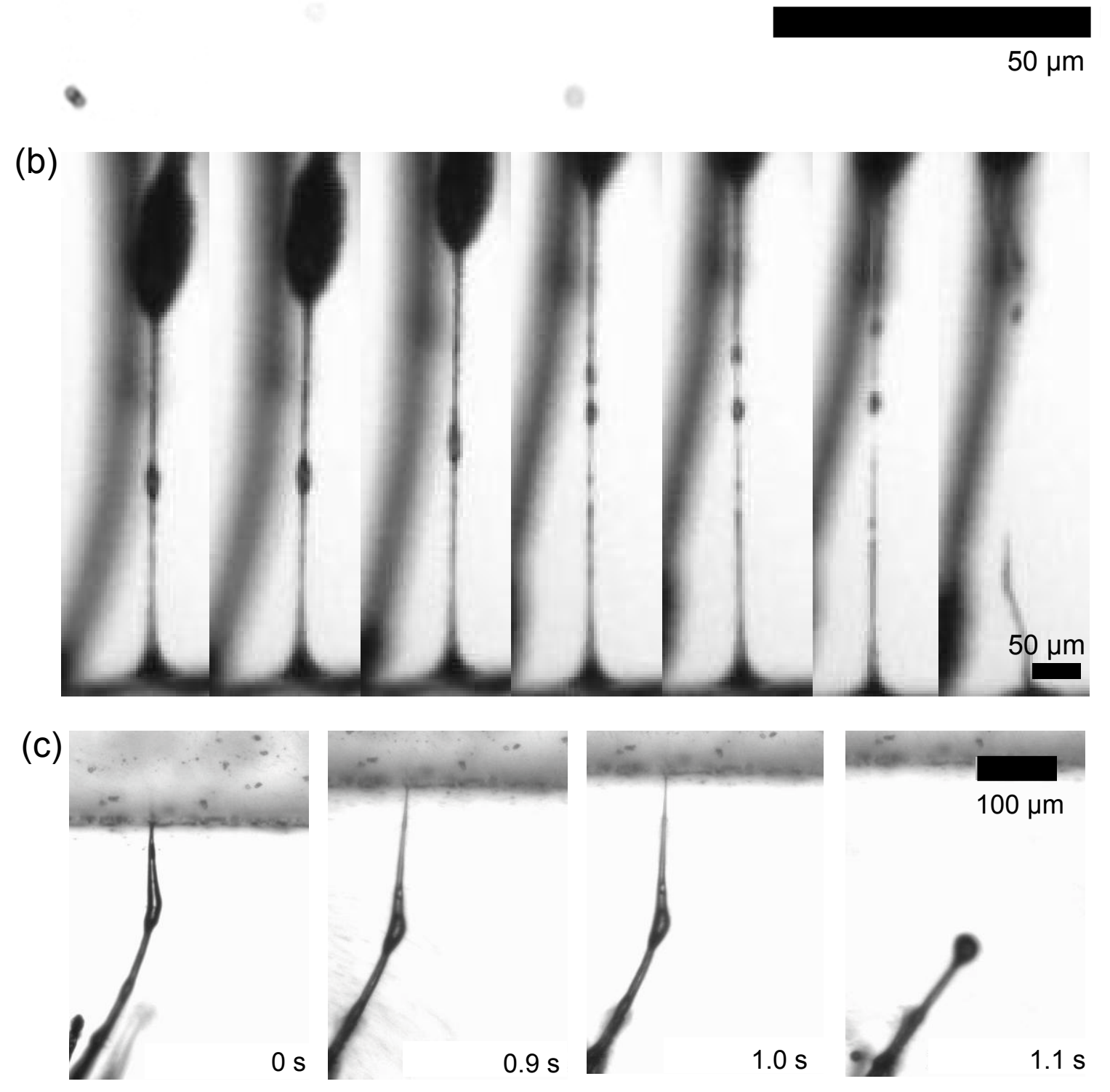

FIG. 8: (a) Examples of the fiber-forming capacity of the mucilage fluid: thread stretched to a final thickness of $R_{\infty} \approx 1.5 \mu \mathrm{m}$. (b) Breakup of mucilage threads: a single bead splits into daughter drops, providing additional extensibility to a drying mucilage thread. Failure occurs only upon further stretching and always within the solidifed, rigid connecting ligaments, but never by elasto-capillary thinning alone; relative humidity $\varphi=0.3$. (c) At high humidity levels $(\varphi=0.8)$, the thread breaks by endplate failure, similar to detachment observed previously upon large deformation stretching of mobile, but strongly strain-stiffening polymer solutions [56]. 